Recepción: 15/01/2019

Aceptación: 19/02/2019

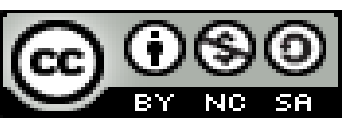

Ciencias económicas y empresariales

Publicación: 05/04/2019

Artículo de investigación

\title{
Créditos cooperativos: Desarrollo para emprendimientos en la comuna Sacachún-Provincia de Santa Elena
}

Cooperative credits: Development for undertakings in the Sacachún-Province of Santa Elena commune

\section{Créditos cooperativos: Desenvolvimento de empreendimentos na comuna de Sacachún-Província de Santa Elena}

\author{
Mesías Heriberto Pilco-Parra ${ }^{\mathrm{I}}$ \\ mesiaspp14@hotmail.com \\ Marcos Antonio Suriaga-Sánchez II \\ marco.suriagas@ug.edu.ec
}

Correspondencia: mesiaspp14@hotmail.com

I Magíster en Administración de Empresas Mención Recursos Humanos y Marketing, Diplomado Superior en Gerencia de Marketing, Especialista en Gerencia de Proyectos, Ingeniero Comercial, Docente Universidad de Guayaquil, Guayaquil, Ecuador.

II Magíster en Administración de Empresas Mención Negocios Internacionales, Magister en Tributación, Contador Público Autorizado, Docente Universidad de Guayaquil, Guayaquil, Ecuador. 


\section{Resumen}

La presente investigación construye un estudio empírico, a través del cual se pretende demostrar la relación entre el financiamiento que otorgan las cooperativas de ahorro y crédito en el desarrollo económico para productores en la comuna Sacachún en la provincia de Santa Elena. Ecuador un país productivo, la población económicamente activa se dedica a esta actividad con demanda de créditos, teniendo 56041 créditos para el año 2016 y una variación positiva de $99 \%$ para el año 2017 según cifras presentadas por el BCE, pero existen impedimentos para el desarrollo, solo accediendo a préstamos grandes y medianos productores, mientras que la área de pequeños productores no cuentan con líneas de crédito, identificando los problemas se puede concluir que existen factores que no han permitido desarrollarse siendo un lugar estratégico de la provincia con suelos que genera buena productividad. Por tanto se analizara el impacto del crédito cooperativo en el desarrollo productivo en la comuna Sacachún de la provincia de Santa Elena, con el fin de proponer alternativas para el aprovechamiento de créditos en el desarrollo, llevando a cabo procesos deductivos centrados en el sector agrícola y los créditos cooperativos, recolectando información documental así como información directamente de productores de las comunas, para analizar las variables obtenidas del grado de relación del desarrollo productivo en el crédito cooperativo.

Palabras clave: Créditos; desarrollo; producción; sector agropecuario.

\section{Abstract}

The present research constructs an empirical study, through which it is tried to demonstrate the relation between the financing that the cooperatives of saving and credit grant in the economic development for producers in the commune Sacachún in the province of Santa Elena. Ecuador a productive country, the economically active population is engaged in this activity with demand for credits, having 56041 credits for 2016 and a positive variation of $99 \%$ for 2017 according to figures presented by the ECB, but there are impediments to development, only by accessing large and medium-sized producers, while the area of small producers does not have credit lines. By identifying the problems, it can be concluded that there are factors that have not allowed to develop being a strategic place in the province with soils that generate good productivity. 
Therefore, the impact of cooperative credit on productive development in the Sacachún commune of the province of Santa Elena will be analyzed, in order to propose alternatives for the use of credits in development, carrying out deductive processes focused on the agricultural sector and the cooperative credits, collecting documentary information as well as information directly from producers of the communes, to analyze the variables obtained from the degree of relationship of the productive development in the cooperative credit.

Keywords: Credits; development; production; agricultural sector.

\section{Resumo}

A presente pesquisa constrói um estudo empírico, através do qual se procura demonstrar a relação entre o financiamento que cooperativas de crédito e poupança concedem ao desenvolvimento econômico de produtores na comuna de Sacachún, na província de Santa Elena. Equador, um país produtivo, a população economicamente ativa está envolvida nessa atividade com demanda por empréstimos, tendo 56041 créditos para 2016 e uma variação positiva de $99 \%$ para 2017 , de acordo com os números apresentados pelo $\mathrm{BCE}$, mas há impedimentos ao desenvolvimento única acesso a empréstimos maiores e médios produtores, enquanto a área de pequenos produtores não têm linhas de crédito, identificação de problemas pode-se concluir que há fatores que têm impedido desenvolvido para ser um lugar estratégico na província com o solo, que gera boa produtividade Assim, analisar-se-á o impacto do crédito cooperativo no desenvolvimento produtivo na comuna de Sacachún, na província de Santa Elena, a fim de propor alternativas para o uso de créditos em desenvolvimento, realizando processos dedutivos voltados para o setor agropecuário e os créditos cooperativos, coletando informações documentais e informações diretamente dos produtores das comunas, para analisar as variáveis obtidas a partir do grau de relação do desenvolvimento produtivo no crédito cooperativo.

Palavras-chave: Créditos; desenvolvimento; produção; setor agrícola 


\section{Introducción}

Este trabajo investigativo de titulación de nivel postgrado, es en referencia al PROYECTO FCIDIPA de la Facultad de Ciencias Administrativas de la Universidad de Guayaquil, relacionado al tema aprobado "Estudio de los emprendimientos exitosos del SALINERITO y propuesta de un modelo de gestión en una comuna de la provincia de Santa Elena" aprobado con resolución: RCU-50-04-121-04-2018.

El presente proyecto de investigación utiliza el método cartesiano, debido a que para su desarrollo se utilizan técnicas de análisis de datos y métodos estadísticos, destinados a comprobar hipótesis que se plantean como parte de este estudio con el fin de comprobar la relación entre variables y plantear soluciones a los problemas identificados

En Ecuador, el Instituto Nacional de Estadísticas y Censos (INEC) ha decidido adaptar la Clasificación Industrial Internacional Uniforme de todas las actividades económicas CIIU para el desarrollo de la Clasificación Nacional de Actividades Económicas, teniendo una gran importancia la agricultura, ganadería, silvicultura y pesca encontrándose en la sección A como se puede identificar en el recuadro. (Servicio de acreditación ecuatoriano, 2017)

Una de las líneas de crédito que opta para la actividad agropecuario es el sector de la Economía Popular y Solidaria (EPS), el cual contribuye al buen vivir, promoviendo a los principios de cooperación, democracia, reciprocidad y solidaridad en las actividades económicas, privilegiando una producción diversificada y eficiente logrando equilibrio entre la producción y el trabajo, poniendo a disposición propuestas de instituciones con modelo social y solidario, dando motivación y potenciación de los productores. (López \& Peñafiel, 2015)

\section{Desarrollo}

Según estudios realizados los recursos financieros han sido canalizados por las Instituciones Financieras de Desarrollo (IFD) agrícola por medio de mecanismos que operan en banca de primer piso y banca de segundo piso, ofertando los créditos de manera directa a los deudores y a través de banca privada comercial y otros intermediarios financieros no bancarios, del estudio realizado a 30 IFD se identificó que 17 son por banca de primer piso mientras que 10 
con banca de segundo piso, y tres optaron por banca de primer y segundo piso.(Banco Interamericano de Desarrollo, 2002, pág. 8).

Con los problemas identificados podemos concluir que existen diversos factores que no han permitido desarrollarse al sector agropecuario como es principalmente el área del sector cooperativo, enfocándonos en Salinas de Guaranda provincia de Bolívar que se sitúa en un lugar estratégico de la provincia por lo que tiene suelos fértiles y productivos obteniendo así una variedad de pisos climáticos y por ende varios tipos de suelos lo que genera productividad agrícola ampliarse en la agricultura y ganadería.(I. Municipalidad de Guaranda -AME , 2015, pág. 11)

El objetivo que se plantea es analizar el impacto del crédito cooperativo en el desarrollo productivo del sector agropecuario en Salinas de Guaranda provincia de Bolívar, con el fin de proponer alternativas para el aprovechamiento de este tipo de créditos en el desarrollo de este sector.

El sector agropecuario en Salinas de Guaranda provincia de Bolívar forma un parte importante en el área de producción tanto agropecuaria como ganadera, pero se da el caso que por la falta de conocimiento impartido comienza a desaparecer el sector agropecuario en Salinas de Guaranda provincia de Bolívar. Además, se puede identificar que las cooperativas prestan buen servicio para el área productiva, teniendo programas que benefician a los productores y hacendados, pero por la falta de educación y conocimiento de los productores optan por no adquirir un préstamo cooperativo y si es para montos altos de dinero prefieren adquirirlo por medio de bancos conocidos, debido a la seguridad que sienten los productores

Transferir herramientas de créditos cooperativos para el sector agropecuario en Salinas de Guaranda provincia de Bolívar en el año 2018 con la finalidad de mejorar la capacidad de generar ingresos, promoviendo a pequeños y medianos productores a un desarrollo continuo, incrementando su producción con productos de excelente calidad teniendo una favorable competencia dentro del mercado.

La presente investigación consiste en un estudio del Ecuador como país productivo enfocado al 
sector agropecuario, teniendo conocimiento que la mayor parte de la población económicamente activa desarrolla esta actividad, por tal motivo se ha creado la economía popular y solidaria siendo una estrategia para el desarrollo y el cambio de la matriz productiva, debido a que aplica principios de cooperativismo donde es beneficiado sectores que se les dificultaba acceder a créditos bancarios, buscando así la participación de sectores más vulnerable ante los requerimientos para su completo progreso.

Por tal motivo la demostración de que el crédito cooperativo para el sector agropecuario ha ayudado al desarrollo y mejoramiento de producción generara más accesibilidad a productores de escasos recursos que han limitado su producción y su desempeño en el mercado

\section{Teoría clásica del crecimiento}

La teoría clásica de crecimiento fue creada por los economistas el siglo XIX, presentado por Adam Smith que veía el crecimiento económico muy limitado por los factores de producción, siendo los rendimientos decrecientes, representa el primer esfuerzo sistemático saldado con relativo éxito por entender los orígenes y las causas de la riqueza de las naciones en su libro del mismo nombre. Smith resaltó el papel de la extensión del mercado para posibilitar la división del trabajo, que a su vez permite la especialización y el incremento de la productividad. En consecuencia, luchó contra el proteccionismo y la excesiva reglamentación de la actividad económica, que interfería en dicha cadena lógica. (Escribano, 2010, pág. 20

Teoría del cooperativismo

Esta teoría es presentada por Louis Blanc La Lega y Mondragón, Louis Jean Joseph Charles Blanc fue un político e historiador socialista francés. Se le considera uno de los precursores de la socialdemocracia, nacido el 29 de octubre de 1811, publicó sus primeros dos volúmenes de la Historie de la Revolution Française. Su publicación fue interrumpida por la Revolución francesa de 1848, en la cual Louis Blanc formó parte del gobierno provisional.

\section{Teoría de la economía solidaria}

Esta teoría fue planteada por el canciller alemán Ludwig Erhard, conocido como el padre de la 
Teoría de la Economía Social de Mercado, quien propició el llamado "milagro alemán", y la Teoría de la Economía Solidaria creada por Luis Razeto Migliaro, las cuales dan fundamento al paradigma socio-económico solidario. (Soto, 2010)

Para el presente estudio de los créditos cooperativos en el desarrollo económico del sector agropecuario del cantón Mejía tendrá un estudio de enfoque mixto, por motivo que se aplicará una investigación cuantitativa y cualitativa. La investigación cualitativa, estudiara la realidad de como suceden hechos que se interpretara deductivamente, utilizando instrumentos para la recolección de información como es la encuesta, obteniendo datos descriptivos tanto del pasado como de la situación actual. Mientras que la investigación cuantitativa se utilizar la recolección de datos por medio del mismo instrumento de recolección de información, con el objeto de analizar los datos, comprobando la hipótesis establecida, por medio de mediciones numéricas, conteo, y el uso de estadística para establecer con exactitud patrones de comportamiento

Se puede mencionar que para un correcto desarrollo del sector agropecuario es necesario obtener líneas de crédito, los cuales fomentan expansión de recursos para un mejor desarrollo económico, debiendo buscar opciones de políticas para mejorar la supervivencia de uno de los sectores más dinámicos y potenciales del país(Revista el agro, 2016).Una de las líneas de crédito que opta para la actividad agropecuario es el sector de la Economía Popular y Solidaria (EPS), el cual contribuye al buen vivir, promoviendo a los principios de cooperación, democracia, reciprocidad y solidaridad en las actividades económicas, privilegiando una producción diversificada y eficiente logrando equilibrio entre la producción y el trabajo, poniendo a disposición propuestas de instituciones con modelo social y solidario, dando motivación y potenciación de los productores.(López \& Peñafiel, 2015)

\section{Metodología}

La presente investigación construye un estudio empírico, a través del cual se pretende demostrar la relación entre el financiamiento que otorgan la Cooperativa de Ahorro y Crédito Salinas (COACSAL) en el desarrollo económico del sector agropecuario en Salinas de Guaranda provincia de Bolívar en los productores caprinos de la comuna de Sacachún en la provincia de Santa Elena. Ecuador un país productivo de sector agropecuario, la población económicamente 
activa se dedica a esta actividad con demanda de créditos.

Investigación documental que se basa en el análisis de la información ya escrita de cómo es el crédito cooperativo y desarrollo productivo del sector agropecuario en los productores caprinos de la comuna de Sacachún en la provincia de Santa Elena.

Investigación de campo debido a que se recolectará información directamente de la fuente como productores del sector agropecuario en los productores caprinos de la comuna de Sacachún en la provincia de Santa Elena. (Bernal, 2010).

Esta investigación es también un estudio insitu ya que se estará en contacto directo en el lugar donde se desarrolla el fenómeno.

También es una investigación de tipo no experimental, por motivo que se analizará el comportamiento de las variables, sin que exista la manipulación de ninguna de las variables, para no alterar el estado natural de las mismas, el cual nos permitirá conocer el grado de relación o asociación que existe entre dos variables dentro de un contexto en particular, y así poder llegar a identificar el grado de relación existente entre varios factores del desarrollo productivo en el crédito cooperativo propuestos dentro de la hipótesis del sector agropecuario en los productores caprinos de la comuna de Sacachún en la provincia de Santa Elena Sampieri, (2010).

\section{Discusión de resultados}

De acuerdo a la investigación realizada se pudo identificar que el sector agropecuario de la comuna Sacachún de la provincia de Santa Elena, forma un parte importante en el área de producción tanto agropecuaria como caprina, ganadera y porcina, pero se da el caso que por la falta de conocimiento impartido comienza a desaparecer el sector agropecuario en la comuna Sacachún.

Además, se puede identificar que las cooperativas prestan buen servicio para el área productiva, teniendo programas que benefician a los productores y hacendados, pero por la falta de educación y conocimiento de los productores optan por no adquirir un préstamo cooperativo y si es para montos altos de dinero prefieren adquirirlo por medio de bancos conocidos, debido a la seguridad que sienten los productores. 


\section{Conclusiones}

Se pudo identificar que las personas que han adquirido un crédito cooperativo han podido experimentar la ayuda del sector cooperativo para el desarrollo productivo en el sector agropecuario en la comuna Sacachún, haciendo crecer su producción y tecnificación en los procesos de producción, para tener mayor oportunidad en el mercado de presentar productos de mejor calidad.

Además, los productores que adquieren prestamos están acostumbrados a pagar mensualmente su crédito, pero la mayor parte de productores termina de cancelar su crédito una vez su producción fue vendida, esta se estima de 6 meses a 1 año, identificando que lo más beneficioso para los productores seria cuotas semestrales, así los productores tendrían una mayor estabilidad económica en el trascurso de su producción.

Tomando en cuenta la importancia que tiene el sector cooperativo para el desarrollo productivo en la comuna Sacachún se recomienda la unión del sector cooperativo de la comuna Sacachún con las otras comunas de la provincia de Santa Elena, a una capacitación de productores sobre créditos enfocados al sector agropecuario, indicando beneficios, tasa de interés, plazos de pago, según estimaciones de producción.

Al momento de adquirir un crédito cooperativo el productor pueda ser guiado para la mejor toma de decisión respecto a plazos de pago, teniendo en cuenta la producción que va a realizar.

Se recomienda al sector cooperativo motivar a los productores por medio de charlas y programas de desarrollo que transmitan el conocimiento agropecuario a sus generaciones, involucrando así a nuevas generaciones para una producción más efectiva y tecnificada.

\section{Referencias Bibliográficas}

Aguirre, R. B. (09 de 6 de 2010). El cooperativismo en el Ecuador. Recuperado el 8 de 10 de 2017

http://www.derechoecuador.com/articulos/detalle/archive/doctrinas/derechocooperativo/2 009/10/26/el-cooperativismo-en-el-ecuador 
Almada, P. (2017). Economía Social. Recuperado el 10 de 10 de 2017, de https://www.econlink.com.ar/economia-social

Anzil, F. (28 de 2 de 2008). Modelo de Solow. Recuperado el 9 de 10 de 2017, de https://www.econlink.com.ar/definicion/solow.shtml

Banco Interamericano de Desarrollo. (7 de marzo de 2002). El papel de los bancos de desarrollo agrícola en el acceso al crédito rural. Recuperado el 7 de 10 de 2017, de Desarrollo de las Economías Rurales en América Latina y el Caribe: https://www.microfinancegateway.org/sites/default/files/mfg-es-documento-el-papel-delos- bancos-de-desarrollo-agricola-en-el-acceso-al-credito-rural-11-2002.pdf

Bernal, C. (2010). Metodología de la Investigación, Tercera edición. Colombia: Pearson Educación.

Castellanos, D. M. (2011). Fórmula para cálculo de la muestra poblaciones finitas. Recuperado el 09 de 10 de 2017, de https://investigacionpediahr.files.wordpress.com/2011/01/formulapara- cc3a1lculo-de-la-muestra-poblaciones-finitas-var-categorica.pdf

Escalante, R., Catalán, H., \& Basurto, S. (2013). Determinantes del crédito en el sector agropecuario mexicano: un análisis mediante un modelo. Recuperado el 1 de 11 de 2017, de Red de Revistas Científicas de América Latina, el Caribe, España y Portugal: http://clacso.redalyc.org/articulo.oa?id=11729145005

Espinosa, E. G., \& Martínez, M. Á. (14 de 02 de 2017). El crédito agropecuario en México. Recuperado el 1 de 11 de 2017, de Red de Revistas Científicas de América Latina y el Caribe, España y Portugal: http://www.redalyc.org/html/2631/263149891013/

INEC. (2010). Proyección de la población ecuatoriana, por años calendario, según cantones. Recuperado el 08 de 10 de 2017, de http://www.ecuadorencifras.gob.ec/documentos/webinec/Poblacion_y_Demografia/Proyecciones_Poblacionales/proyeccion_cantonal_total_2 010- 2020.xlsx

INEC. (Junio de 2012). Clasificación Nacional de Actividades Económicas. Recuperado el 04 de 10 de 2017, de http://aplicaciones2.ecuadorencifras.gob.ec/SIN/descargas/ciiu.pdf

Instituto Espacial Ecuatoriano IEE. (Septiembre de 2013). Memoria técnica. Recuperado el 03 de 10 de 2017, de generación de geo información para la gestión del territorio a nivel 
nacional:

http://app.sni.gob.ec/sni-

link/sni/pdot/zona2/nivel_del_pdot_cantonal/pichincha/mejia/iee/memoria_tecnicA/mt_m ejia_socioeconomico.pdf

Pellini, C. (2017). El Cooperativismo: Principios, Concepto - Definición Cooperativas. Recuperado el 10 de 10 de 2017, de https://historiaybiografias.com/conceptos12/

Servicio de acreditación ecuatoriano. (10 de 02 de 2017). ¿Qué son los códigos CIIU? Recuperado el 04 de 10 de 2017, de http:/www.acreditacion.gob.ec/que-son-los-codigosciiu/

Trigo, E. (19 de 5 de 2008). Desarrollo Agrícola. Recuperado el 10 de 10 de 2017, de http://desarrolloagricolajb.blogspot.com/

Zabala, H. (2016). Economía agraria y asociatividad cooperativa en Colombia. Recuperado el 10 de 10 de 2017, de

http://www.funlam.edu.co/uploads/fondoeditorial/230_Economia_agraria_y_asociatividad_coo perativa_en_Colombia.pdf 